\title{
Crack localization in a double-punched concrete cuboid with time reverse modeling of acoustic emissions
}

\author{
Georg Karl Kocur • Thomas Vogel • \\ Erik H. Saenger
}

Received: 17 December 2010 / Accepted: 21 July 2011 / Published online: 7 September 2011

(C) Springer Science+Business Media B.V. 2011

\begin{abstract}
Time reverse modeling (TRM) is successfully applied to localize acoustic emissions (AE) obtained from a physical experiment (double punch test) on a $118 \times 120 \times 160 \mathrm{~mm}$ concrete cuboid. Previously, feasibility studies using numerical (Ricker wavelet) and experimental (pencil-lead break) excitations are performed to demonstrate the applicability of TRM to real AE waveforms. Numerical simulations are performed assuming an uncracked and heterogeneous concrete model. The localization results from the numerical and experimental feasibility studies are compared and verified. The AE recorded during the double punch test are localized in a three-dimensional domain using TRM. The localization results are superposed with the three-dimensional threshold-segmented crack patterns obtained from X-ray computed tomography scans of the failed concrete cuboid. The presented TRM approach represents a reliable localization tool for signal-based $\mathrm{AE}$ analysis.
\end{abstract}

\footnotetext{
G. K. Kocur $(\varangle) \cdot$ T. Vogel

Institute of Structural Engineering, ETH Zurich,

Wolfgang-Pauli-Strasse 15, 8093 Zurich, Switzerland

e-mail: kocur@ibk.baug.ethz.ch

T. Vogel

e-mail: vogel@ibk.baug.ethz.ch

E. H. Saenger

Geological Institute, ETH Zurich, Sonneggstrasse 5,

8092 Zurich, Switzerland

e-mail: erik.saenger@erdw.ethz.ch;

erik.saenger@spectraseis.com
}

Keywords Time reverse modeling . Elastic wave propagation - Non-destructive testing . Acoustic emission · Concrete cracking

\section{Introduction}

The numerical simulation of elastic wave propagation is a powerful method for visualizing the elastic energy flow and distribution in infinite and semi-infinite continua. A possible application in the area of nondestructive concrete testing is the numerical modeling of acoustic emission (AE). AE is defined as a spontaneous release of strain energy caused by irreversible processes such as cracking or internal friction, which result in elastic waves propagating in a structure. The emitted waves are recorded as normal displacements on the specimens' surfaces using piezoelectric sensors. They are converted to voltage signals, which are referred to as acoustic emission [see for example Grosse and Ohtsu (2008)].

There are two core areas in acoustic emission analysis: parameter-based and signal-based techniques. In the parameter-based techniques certain parameters are sought to find in the AE wave packets. Some essential parameters are hits, amplitude and wave energy. Their occurrence and accumulated trends are of particular interest for qualitative $\mathrm{AE}$ analysis (Kocur and Vogel 2010). In signal-based AE techniques the complete waveform is considered. A common application is the determination (picking) of the arrival (onset) time 
of the P-wave motion. Together with a homogeneous velocity model, the respective three-dimensional $\mathrm{AE}$ source coordinates can be calculated using a localization algorithm (Kurz et al. 2005). The method considered to be the most reliable is presented by Schechinger and Vogel (2007). In this method, AE sources are localized based on iteratively calculated onset times and a heterogeneous velocity model. Due to the overdetermined system of linear equations, additional error estimations can be performed as well.

Fink et al. (2000) presented an alternative approach, the so-called time reverse modeling (TRM), in which the time-reversals of ultrasonic fields are used to focus on sources through an inhomogeneous medium. An array of sensors capable of emitting and transmitting pressure fields is used as a time-reversal mirror. The emitted pressure field is time-reversed and re-emitted. The resulting dominant concentration of the wavefield represents the position of its origin. Steiner et al. (2008) applied TRM in the area of exploration geophysics, where this technique is used to localize hydrocarbon reservoirs. Presently, the TRM approach is being adopted in the field of non-destructive testing (Saenger et al. 2011) and applied to concrete. Numerical studies have been performed to localize AE-like sources in a heterogeneous concrete model. The results provide valuable information that is applied in the present research.

In the present study, AE waveforms obtained from a double punch test on a concrete specimen $(120 \times 118 \times$ $160 \mathrm{~mm}$ ) are used to numerically simulate elastic wave propagation. To verify the accuracy of TRM, numerical tests are carried out, for excitation sources that are either obtained numerically or experimentally. TRM uses numerical algorithms for elastic wave propagation in combination with an imaging condition. Elastic waves emitted from a real AE source such as a pencillead break are recorded by piezoelectric sensors on the specimens' surfaces. Alternatively, a synthetic excitation source generated numerically and the corresponding calculated displacements at the original sensor positions can be used. The effective elastic properties such as the compression and shear wave velocities are calculated assuming a heterogeneous medium. The medium is represented by a numerical concrete model (NCM) with concrete constituents randomly distributed in space, as outlined in Schubert and Schechinger (2002). The resulting effective velocity distribution is a major part of the model used in TRM. The time reverse computation is carried out for both the measured AE waveforms and the numerically computed displacements. In both cases, active sources at the original sensor positions send the complete waves back in time using the previously determined velocity model. The induced wavefronts propagate and subsequently concentrate on the source location.

\section{Physical experiment}

\subsection{Preliminary considerations}

For the physical experiment, the test setup of the classic double punch test according to Chen (1970) is adopted. Because the sensors can be mounted more easily on a plane surface, a cuboid is used instead of a cylinder. Originally, the test was developed for determining the concrete tensile strength $f_{c t}$, which can be approximated by the formula $F_{u} /\left[\pi\left(1.2 b h-p^{2}\right)\right]$; more details can be found in Chen (1970) and Marti (1989). A concrete cylinder of height $h(160 \mathrm{~mm})$ and diameter $2 b$ $(120 \mathrm{~mm})$ is compressed by two steel punches of diameter $2 p(30 \mathrm{~mm})$, located concentrically on its top and bottom surfaces (see dotted line in Fig. 1a). When the ultimate load $F_{u}(106.4 \mathrm{kN})$ is reached the cylinder splits radially. Sliding takes place at the cone surfaces while separation occurs at the radial crack surfaces between the outer segments (see dashed line in Fig. 1b). The greatest amount of fracture energy is released due to the separation failure. The predictable crack pattern that develops is used in this paper to check the accuracy of the TRM localization procedure.

\subsection{Experimental setup}

A $120 \times 118 \times 160 \mathrm{~mm}$ cuboid is compressed to failure by two $30 \mathrm{~mm}$ diameter steel punches. Acoustic emissions are recorded by 8 piezoelectric sensors which are arranged irregularly on the specimens' surfaces at the coordinates shown in Table 1. The piezoelectric sensors are clamped to the surfaces. A thin layer of grease is applied to the sensors to ensure ideal coupling. Before measurement of $\mathrm{AE}$, the sensors are relatively calibrated with pencil-lead breaks. A commercial AE recording system (AMSY5, Vallen-Systeme) with transient piezoelectric sensors (KSB250, Ziegler Instruments), sensitive to a bandwidth of $50-250 \mathrm{kHz}$, is 
(a)

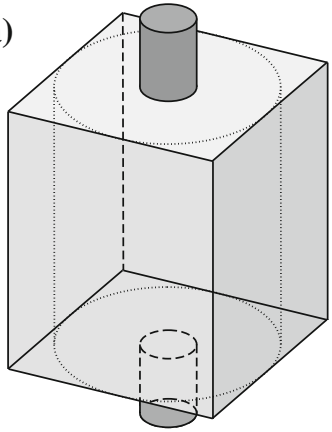

(b)

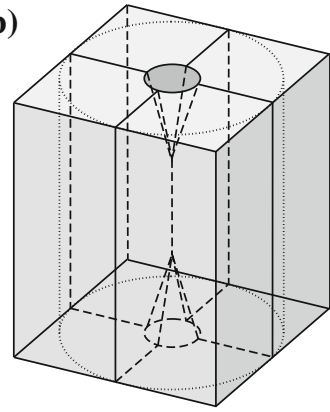

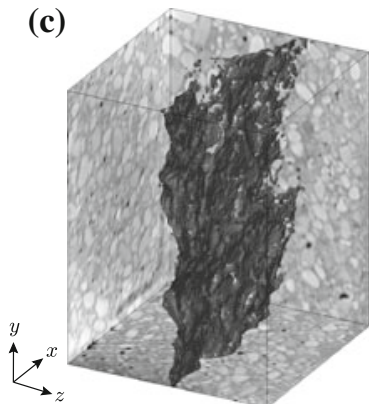

Fig. 1 Setup of a double punch test carried out on a cuboid (a), schematic illustration of the failure mechanism according to Chen (1970) (b), superposition of the projected X-ray computed

Table 1 Coordinates of the sensor positions on the concrete cuboid

\begin{tabular}{lrrr}
\hline Sensor & $x(\mathrm{~mm})$ & $y(\mathrm{~mm})$ & $z(\mathrm{~mm})$ \\
\hline$\# 1$ & 25 & 160 & 30 \\
$\# 2$ & 30 & 95 & 0 \\
$\# 3$ & 100 & 20 & 0 \\
$\# 4$ & 120 & 135 & 30 \\
$\# 5$ & 120 & 30 & 90 \\
$\# 6$ & 100 & 100 & 118 \\
$\# 7$ & 30 & 25 & 118 \\
$\# 8$ & 0 & 40 & 35 \\
\hline
\end{tabular}

used. The sensors dominantly respond to wave motion normal to the surface. The signals are preamplified (AEP4, Vallen Systeme) with a gain of $40 \mathrm{~dB}$. During the entire loading history, complete waveforms are recorded for a duration $t=409.6 \mu \mathrm{s}$ and with a sampling frequency $f_{s}=10 \mathrm{MHz}$. The load is applied in displacement-controlled increments of $0.004 \mathrm{~mm} / \mathrm{s}$ until separation failure is reached. The corresponding load-deformation curve can be seen in Fig. 2. After loading and the $\mathrm{AE}$ measurements, the failed cuboid is stabilized and scanned with X-rays to determine the inner crack pattern. For $\mathrm{AE}$ analysis, four $\mathrm{AE}$ sets (TR_75, TR_82, TR_84 and TR_180) are used, selected in view of a low noise-to-amplitude ratio. The AE sets TR_75-84 occur consecutively at a load level between 48.3 and $51.2 \mathrm{kN}$, whereas TR_180 occurs at a load of $63.3 \mathrm{kN}$. No correlation between the selected $\mathrm{AE}$ sets and the decays in the load can be observed in Fig. 2. tomography (CT) images and the threshold-segmented separating crack after failure (c). The approximate size of the cuboid is $120 \times 118 \times 160 \mathrm{~mm}(x \times z \times y)$

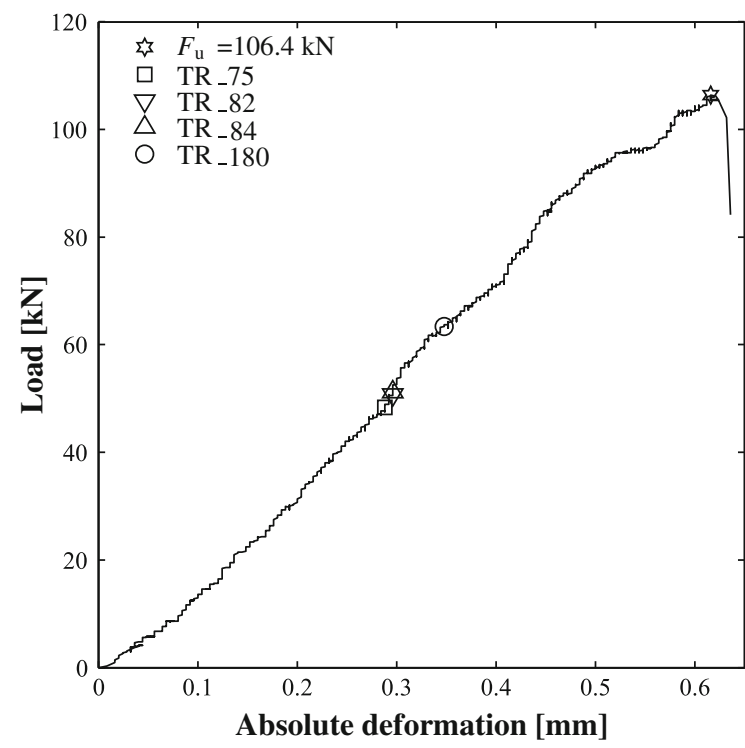

Fig. 2 Load-deformation curve of the double punch test with the four selected AE sets marked

\subsection{X-ray computed tomography scan of the specimen after failure}

The X-ray computed tomography (CT) scans are carried out with a Dual-Source CT scanner provided by the Institute of Diagnostic Radiology of the University of Zurich. The X-ray CT parameters used for the scans are $140 \mathrm{kV}$ and $350 \mathrm{mAs}$ providing a voxel size of $254 \mu \mathrm{m}^{3}(6.3 \times 6.3 \times 6.3 \mu \mathrm{m})$. From the X-ray CT scanner a sequence of $\mathrm{X}$-ray slice data is obtained and post-processed (threshold-segmented). During the segmentation process, the crack volume is separated using 


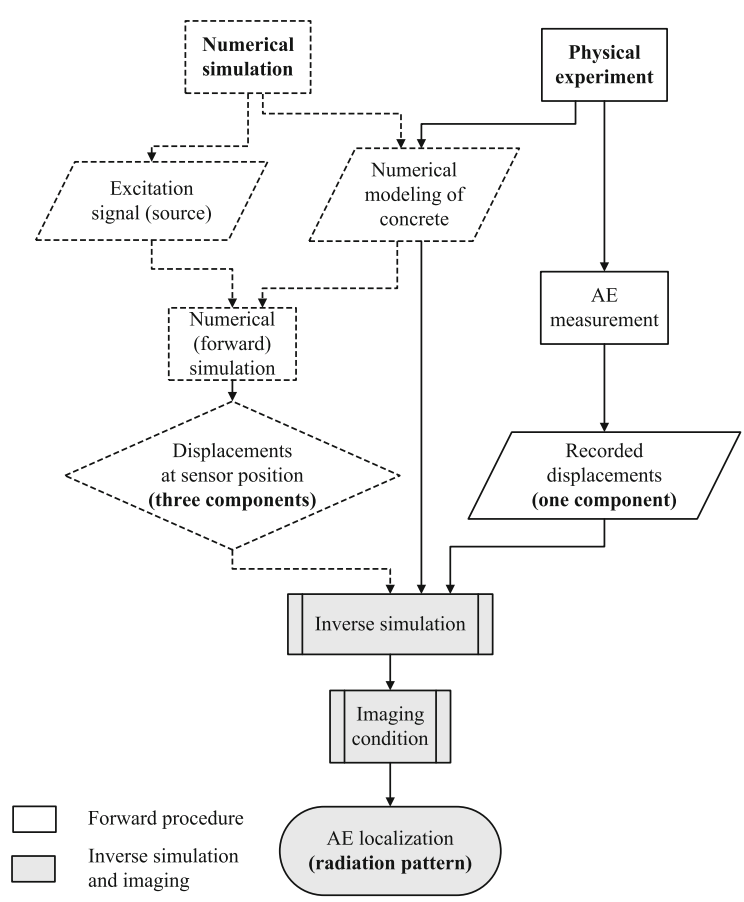

Fig. 3 Flow chart showing the intermediate steps of the TRM procedure for concrete

a lower and upper threshold limit of the mean radiodensity of air [-1,024;745], specified on the so-called Hounsfield scale in Hounsfield units (HU). Details of the post-processing procedure can be found in Kocur et al. (2010) for similar specimens. The separating crack, which splits the concrete specimen into two parts, can be seen in Fig. 1c.

\section{Time reverse modeling in concrete}

\subsection{Principle}

TRM is based on ultrasonic wavefields that are reversed in time and re-emitted numerically into the specimen, where they concentrate on the location of the original source (Fink et al. 2000). The procedure consists of two steps: the forward procedure and inverse simulation including imaging (see Fig. 3). The forward procedure provides the input signal, either numerically, with full control of its origin, or experimentally, obtained for example from acoustic emission measurements. In the numerical forward simulation, a time dependent excitation is applied at a spatial point in the model. Displacements at sensor positions at the model's boundaries are calculated over time. Dealing with a complex and strongly heterogeneous material such as concrete, a representative numerical model needs to be established as a basis for further numerical simulations. In physical experiments such as the punching test, concrete cracking releases stored strain energy, and the associated elastic waves propagate through the medium. The resulting time-dependent displacements at the sensor positions, determined either numerically or experimentally, serve as input signals for subsequent TRM simulations. A feasibility study on a two-dimensional concrete model in Saenger et al. (2011) demonstrates that a few boundary points, equivalent to 12 sensors, are sufficient to achieve good results in the localization of purely numerical excitations.

\subsection{Forward simulation in concrete}

\subsubsection{Elastic wave propagation}

The spatial domain of interest is denoted by $\Omega$ with the positions $\mathbf{x} \in \Omega$. The time $t$ is defined in $[0 ; T]$ with end time $T$. The displacement field $u_{i}=\mathbf{u}(\mathbf{x}, t) \in \mathbb{R}^{3}$ fulfills the wave equations for the linear-elastic continuum

$\rho u_{i, t t}=\sigma_{i j, j}+f_{i} \quad$ in $\quad \Omega \times[0 ; T]$

where $\rho=\boldsymbol{\rho}(\mathbf{x})$ denotes a space-dependent density and $f_{i}=\mathbf{f}(\mathbf{x}, t)$ denotes a space- and time-dependent body force. The summation convention is implied. Applying Hooke's law $\sigma_{i j}=C_{i j k l} \varepsilon_{k l}$, where $\sigma_{i j}$ and $\varepsilon_{i j}$ are the stress and strain tensors, respectively, and $C_{i j k l}$ is a fourth-order elastic tensor, Eq. (1) can be expanded to

$\rho u_{i, t t}=(\lambda+\mu) u_{k, k i}+\mu u_{i, k k}+f_{i}=\sigma_{i j, j}+f_{i}$

and written in vector notation

$\rho \ddot{\mathbf{u}}=(\lambda+\mu) \nabla \nabla \cdot \mathbf{u}+\mu \nabla^{2} \mathbf{u}+\mathbf{f}=\nabla \cdot \sigma+\mathbf{f}$

with Lamé's constants $\lambda$ and $\mu$ (Achenbach 1973). The Lamé constants $\lambda$ and $\mu$ are connected to the $\mathrm{P}$-wave velocity $c_{p}=\sqrt{(\lambda+2 \mu) / \rho}$ and $S$-wave velocity $c_{s}=\sqrt{\mu / \rho}$. Equation(2) is used in the forward (numerical) simulation to calculate the displacements at the sensor positions on the domain's boundary $\partial \Omega$. The wave equation is discretized with the so-called rotated staggered-grid finite-difference scheme described in Saenger et al. (2000). 
Fig. 4 The three phases of concrete are used in the three-dimensional NCM to model the material behavior of a $100 \times 100 \times 100 \mathrm{~mm}$ concrete cube, discretized with $400 \times 400 \times 400$ grid points: aggregate (a), cement matrix (b) and air voids (c)

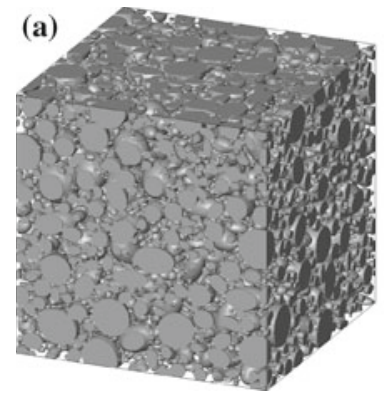

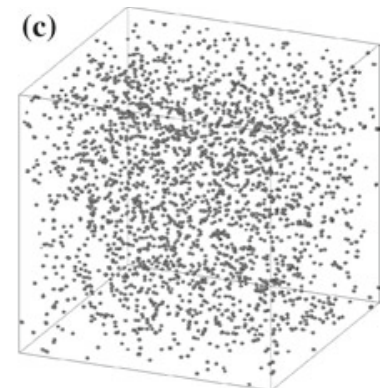

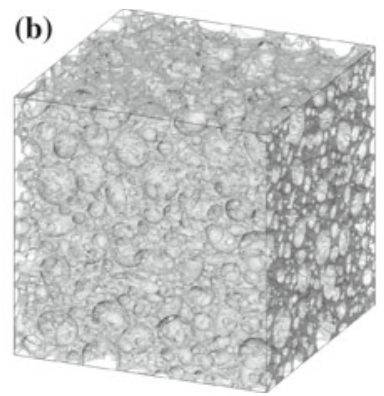

\subsubsection{Numerical concrete model}

For the simulation of elastic wave propagation in concrete, the components of $C_{i j k l}$ need to be approximated realistically. A numerical concrete model (NCM) is therefore established. In the NCM three phases are superimposed: aggregate (Fig. 4a), cement matrix (Fig. 4b) and air voids (Fig. 4c). The geometries of the concrete constituents are simplified using basic shapes such as ellipsoids (aggregate grains) and spheres (air voids), which are randomly distributed in space as suggested by Schubert and Schechinger (2002). More complex approximations for particle shapes such as presented in Häfner et al. (2006) are not deemed necessary. This refinement provides little additional information for the simulation of wave propagation for a governing wavelength of $\Lambda \cong 4.0 \mathrm{~cm}$ $\left(c_{p \text {,eff }} \cong 4,000 \mathrm{~m} / \mathrm{s}\right.$ and $\left.f_{c}=100 \mathrm{kHz}\right)$. The volume of air voids in concrete is estimated at $1 \%$, and a constant void diameter of $2 \mathrm{~mm}$ is conservatively assumed. Wave velocities $c_{p}$ and $c_{s}$ and the density $\rho$ of each grain vary within realistic ranges. The aggregate grains are assumed to have a maximum diameter of $16 \mathrm{~mm}$. The remaining volume is filled with a homogeneous cement matrix. The concrete mix matches Fuller's curve in Fuller and Thomson (1907). The material properties used for generating the NCM are summarized in Table 2. The NCM and its associated assumptions are verified experimentally and numerically in Kocur et al. (2010).

\subsubsection{Effective elastic properties}

Once the numerical model for concrete is established, the effective elastic properties (EEP) such as $c_{p, \text { eff }}, c_{s, \text { eff }}$ and $\rho_{\text {eff }}$ are determined following

the dynamic wave propagation method presented by Saenger (2008). The aim of this approach is to study the speed of elastic waves in heterogeneous materials, herein represented by the NCM, in the long wavelength range. The NCM is embedded between two layers, one at the top and one at the bottom. A plane body forcethe excitation-is applied to the top of the model. The source wavelet is the first derivative of a Gaussian with a central frequency $f_{c}=12.5 \mathrm{kHz}$ and a time increment $\Delta t=1.8 \times 10^{-8} \mathrm{~s}$. Two horizontal planes of sensors are located at the top and bottom of the model, respectively. The time delay of the mean-peak amplitude of the plane wave that emerges between the top and bottom is used to estimate the effective velocities $c_{p \text {, eff }}$ and $c_{s, \text { eff }}$. The effective density $\rho_{\text {eff }}$ is determined by averaging the density of all grid points over the volume. For all numerical simulations the concrete is assumed to be uncracked. As reported in Kocur et al. (2010), the contrast between the acoustic impedances of aggregate and cement paste is marginal for the governing wavelength $(\Lambda \cong 4.0 \mathrm{~cm})$. The air voids have a scattering effect on the propagating wavefront, which reduces the EEP. For the numerical simulations, a representative homogeneous density and velocity distribution is approximated by the calculated EEP. The EEP of the presented NCM are listed in Table 2.

\subsection{Inverse simulation}

The forward simulation provides time series of the displacements (Fig. 5)

$$
\mathbf{u}^{(k)}(t)=\mathbf{u}\left(\mathbf{x}^{(k)}, t\right)
$$

at positions $\mathbf{x}^{(k)}$ of the sensor locations

$S=\left\{\mathbf{x}^{(1)}, \mathbf{x}^{(2)}, \ldots, \mathbf{x}^{(N)}\right\} \subset \partial \Omega$. 
Table 2 Material parameters adopted from Kocur et al. (2010), used to generate the three-dimensional numerical concrete model and the calculated effective elastic parameters $(*)$

\begin{tabular}{lllll}
\hline Property (unit) & Aggregate & Cement & Air voids & ${ }^{*}$ EEP \\
\hline$c_{p}\left({ }^{*} c_{p, \text { eff }}\right)(\mathrm{m} / \mathrm{s})$ & $4,180 \pm 210$ & 3,950 & 0 & 3,987 \\
$c_{s}\left({ }^{*} c_{s, \text { eff }}\right)(\mathrm{m} / \mathrm{s})$ & $2,475 \pm 125$ & 2,250 & 0 & 2,328 \\
$\rho\left({ }^{*} \rho_{\text {eff }}\right)\left(\mathrm{kg} / \mathrm{m}^{3}\right)$ & $2,610 \pm 130$ & 2,050 & 0.0001 & 2,200 \\
\hline
\end{tabular}

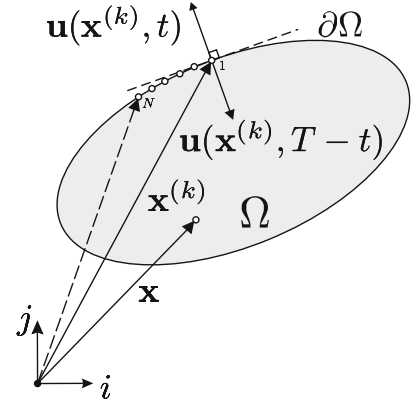

Fig. 5 Definition of the TRM domain

$N$ denotes the number of sensors that are used for the simulation. The inverse simulation is performed for velocities $c_{p \text {,eff }}$ and $c_{s, \text { eff }}$ and the density $\rho_{\text {eff }}$, which were previously determined in the numerical forward simulation. The body force $f_{i}$ in Eq. (2) is set to zero. The recorded displacements $\mathbf{u}^{(k)}(t)=\left[u_{x}^{(k)}(t), u_{y}^{(k)}(t), u_{z}^{(k)}(t)\right]$ on the boundary $\partial \Omega$ are fed into the domain as excitation sources. Formally the inverse displacement field is denoted as

$\mathbf{u}^{(k)}(t)=\mathbf{u}\left(\mathbf{x}^{(k)}, T-t\right)$ for $\mathbf{x} \in S \subset \partial \Omega$.

\subsection{Imaging conditions}

The re-emitted wavefields will interfere with each other for $t \in[0 ; T]$. In the later stages of the simulation the interferences cannot be identified unless imaging conditions are applied. Four imaging conditions are used to handle this problem: the maximum particle displacement $\mathbf{u}_{\max }(\mathbf{x})$, the two maximum densities for separated $\mathrm{P}$ - and $\mathrm{S}$-wave energy parts $\mathbf{E}_{\mathrm{p}}(\mathbf{x})$ and $\mathbf{E}_{\mathrm{S}}(\mathbf{x})$, and the maximum total energy density $\mathbf{E}_{\text {tot }}(\mathbf{x})$ (see Fig. 6). Note that the relation $\mathbf{E}_{\text {tot }}(\mathbf{x})=\mathbf{E}_{\mathrm{p}}(\mathbf{x})+\mathbf{E}_{\mathrm{s}}(\mathbf{x})$ is valid for wave propagation in a homogeneous and unbounded medium in the far field.

\subsubsection{Maximum particle displacement}

Steiner et al. (2008) introduced the idea of taking only the maximum absolute value of the particle displacement into account, which is defined as

$$
\mathbf{u}_{\max }(\mathbf{x}):=\max _{t \in[0 ; T]}\|\mathbf{u}(\mathbf{x}, T-t)\| \text {. }
$$

The maximum particle displacement $\mathbf{u}$ for every point $\mathbf{x}$ on $\Omega$ of the inverse wavefield is stored for the entire time $t \in[0 ; T]$ of modeling.
Fig. 6 Imaging conditions according to Morse and Feshbach (1953) for the far-field term of the displacement field in a homogeneous and unbounded medium

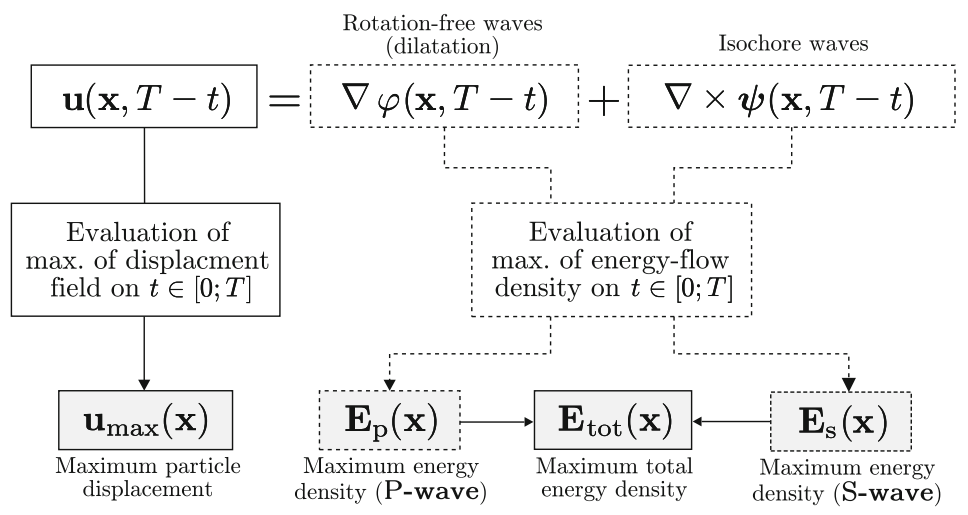




\subsubsection{Maximum energy density}

If the inverse wavefield $\mathbf{u}(\mathbf{x}, T-t)$ is separated into rotation-free $(\nabla \varphi(\mathbf{x}, T-t)=0)$ and isochore parts $(\nabla \times \psi(\mathbf{x}, T-t)=0)$, then two further imaging conditions can be derived as shown by Morse and Feshbach (1953) and Steiner et al. (2008): the maximum energy density of the P-wave

$\mathbf{E}_{\mathrm{p}}(\mathbf{x}):=\max _{t \in[0 ; T]}(\lambda+2 \mu)[\nabla \cdot \mathbf{u}(\mathbf{x}, T-t)]^{2}$

and the $\mathrm{S}$-wave

$\mathbf{E}_{\mathrm{S}}(\mathbf{x}):=\max _{t \in[0 ; T]} \mu[\nabla \times \mathbf{u}(\mathbf{x}, T-t)]^{2}$.

Due to some limitations of these conditions, Saenger (2011) proposed a more promising imaging condition of maximum total energy density:

$\mathbf{E}_{\mathrm{tot}}(\mathbf{x}):=\max _{t \in[0 ; T]}\{\boldsymbol{\sigma}(\mathbf{x}, T-t) \boldsymbol{\varepsilon}(\mathbf{x}, T-t)\}$.

The maximum total energy density at any point on domain $\Omega$ is the scalar product of the stress tensor $\sigma_{i j}=\boldsymbol{\sigma}(\mathbf{x}, T-t)$ and the strain tensor $\varepsilon_{i j}=\boldsymbol{\varepsilon}(\mathbf{x}, T-t)$ for the entire modeling time $t \in[0 ; T]$ at every point of domain $\Omega$.

\section{Inverse simulation with acoustic emission}

\subsection{Feasibility study on TRM of acoustic emission in concrete}

\subsubsection{Purely numerical excitation}

In this section, a feasibility study of TRM applied to the three-dimensional NCM is carried out. As the AE equipment at our disposal consists of 8 sensors capable of recording displacements normal to the surface, the numerical forward simulation involves $N=8$ sensor locations $\mathbf{x}^{(1, \ldots, N)}$, at which the calculated displacements $\mathbf{u}\left(\mathbf{x}^{(1, \ldots, N)}, t\right)$ normal to $\partial \Omega$ are recorded (see Fig. 5). The excitation signal

$h(t)=-2 \pi^{2} f_{c}^{2}\left(t-t_{0}\right)^{2} e^{-\pi^{2} f_{c}^{2}\left(t-t_{0}\right)^{2}}$

is a Ricker2 wavelet, which is the second derivative of the Gaussian function expressed in terms of the central frequency $f_{c}$. The numerical forward simulation is performed with a sampling frequency $f_{s}=10 \mathrm{MHz}$ for a duration of $t=409.6 \mu \mathrm{s}$. The Ricker2 wavelet is induced at 15 grid points distance from Sensor\#4. The $\mathrm{NCM}$ is used for the simulation, with a grid spacing
$\Delta h=0.001 \mathrm{~m}$. A central frequency $f_{c}=100 \mathrm{kHz}$ and a time increment $\Delta t=5.0 \times 10^{-9} \mathrm{~s}$ are used. The model with $120 \times 118 \times 164$ grid points $(\mathrm{gp})(\mathrm{gp}$ is equivalent to $\mathrm{mm}$ ) is implemented with a rotated staggered-grid scheme as suggested by Saenger et al. (2000), using the finite difference method. The inverse simulation is performed for a duration of $t=600 \mu \mathrm{s}$, which allows for sufficient interference of the complete wavefields. After applying the imaging condition from Eq. (10) and cutting a slice of the scalar energy field at $y=135 \mathrm{~mm}$, two dominant energy concentrations at Sensor\#4 (Fig. 7a) can be observed.

In general, the localization with TRM works well for the purely numerical example. The location of the source can be identified in Fig. 7a. On a critical note, the energy radiated by the sensors themselves may interfere with the focus of the source, regardless of which imaging conditions in Eqs. (7)-(10) are applied. This is the case for example if the source is located too close to a sensor. To eliminate the sensor's energy radiation, an intermediate step is suggested by Witten and Artman (2011). The stored displacements $\mathbf{u}^{(k)}(t)=\left[u_{x}^{(k)}(t)\right], \mathbf{u}^{(k)}(t)=\left[u_{y}^{(k)}(t)\right]$ or $\mathbf{u}^{(k)}(t)=$ $\left[u_{z}^{(k)}(t)\right]$ (depending on the orientation of the respective sensor at the sample surface) are numerically re-emitted into the system without being reversed in time. The corresponding scalar energy field, denoted as $\mathbf{E}_{\text {tot }}(\mathbf{u}(\mathbf{x}, t))$ can be seen in Fig. 7b. Note that to avoid confusion in the following sections, $\mathbf{E}_{\text {tot }}(\mathbf{x})=$ $\mathbf{E}_{\text {tot }}(\mathbf{u}(\mathbf{x}, T-t))$ is used to denote the time-reversed energy field. The ratio of both scalar fields should theoretically exclude energy radiation from the sensors. The new imaging condition is formally written as

$\hat{\mathbf{E}}_{\text {tot }}(\mathbf{x}):=\frac{\mathbf{E}_{\text {tot }}(\mathbf{u}(\mathbf{x}, T-t))}{\mathbf{E}_{\text {tot }}(\mathbf{u}(\mathbf{x}, t))}$

and referred to as the related maximum total energy density. In Fig. 7c, the resulting scalar energy field at $y=135 \mathrm{~mm}$ can be seen, where the source is now readily identifiable. Having demonstrated a successful TRM localization in concrete for a numerical excitation source, in the next section real $\mathrm{AE}$ waveforms from pencil-lead breaks are applied instead of numerical excitations.

\subsubsection{Excitation by pencil-lead break}

Three physical experiments are carried out. The coupling of all sensors is tested by breaking a pencil-lead, 

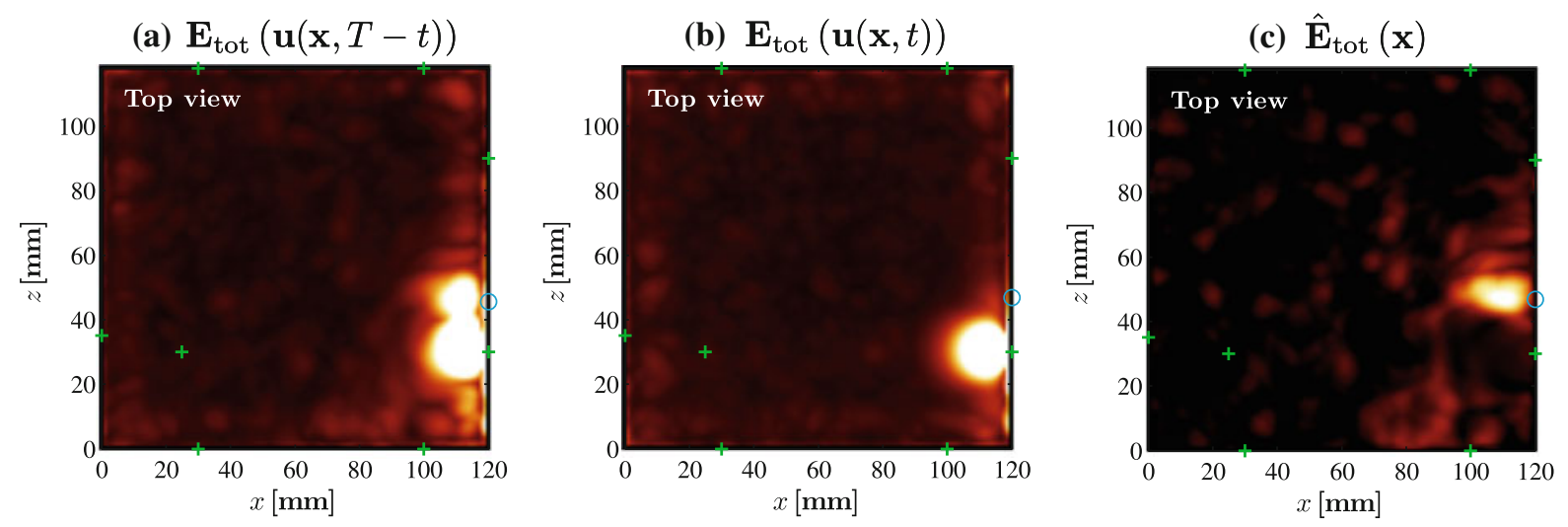

Fig. 7 Specimen cross-sections at $y=135 \mathrm{~mm}$ illustrating the maximum total energy densities: (a) $\mathbf{E}_{\text {tot }}(\mathbf{u}(\mathbf{x}, T-t))$, (b) $\mathbf{E}_{\text {tot }}(\mathbf{u}(\mathbf{x}, t))$ and $(\mathbf{c}) \hat{\mathbf{E}}_{\text {tot }}(\mathbf{u}(\mathbf{x}, t))=\frac{\mathbf{E}_{\mathrm{tot}}(\mathbf{u}(\mathbf{x}, T-t))}{\mathbf{E}_{\mathrm{tot}}(\mathbf{u}(\mathbf{x}, t))}$. The blue circles indicate the numerical Ricker 2 excitation sources located 15 gp away from Sensor \#4 at $[x, z, y]=[120,45,135] \mathrm{mm}$. The green crosses represent the sensor positions (a) Sensor\#3

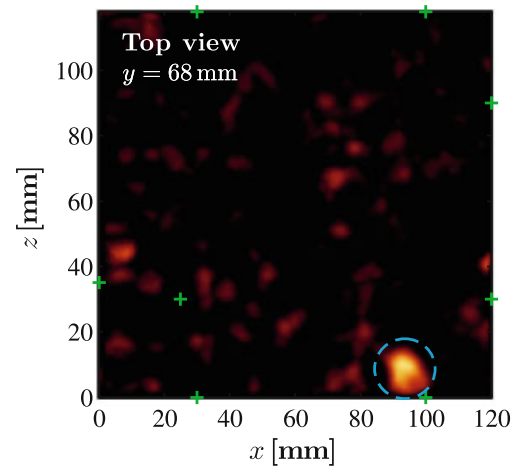

(b) Sensor \#6

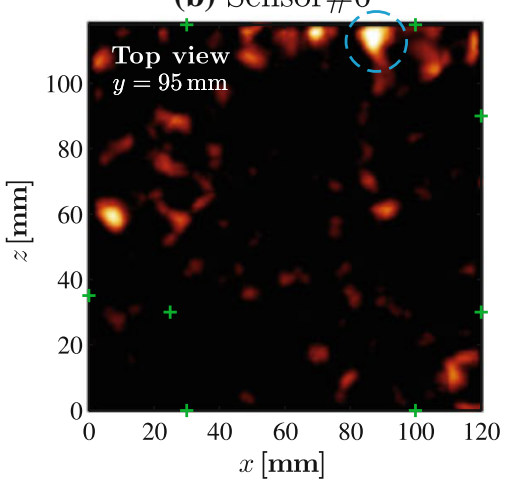

(c) Sensor \#7

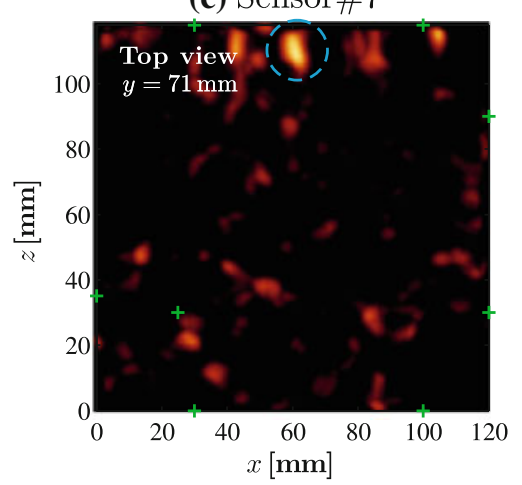

Fig. 8 Specimen cross-sections illustrating the related maximum total energy density $\hat{\mathbf{E}}_{\text {tot }}(\mathbf{x})$ at: (a) $y=68 \mathrm{~mm}$, (b) $y=$ $95 \mathrm{~mm}$ and (c) $y=71 \mathrm{~mm}$. Dominant energy concentrations due

also known as the Hsu-Nielsen source (Grosse and Ohtsu 2008), at a distance of approx. $10 \mathrm{~mm}$ from the respective sensor. Each break provides a set of transient signals, which can be used as input for the inverse simulation. The normal displacements recorded by the piezoelectric sensors at the sample surface by the piezoelectric sensors are stored, time reversed and numerically re-emitted into the system. The previously determined EEP of concrete are used for the numerical model of the inverse simulation. The duration times of the recorded $\mathrm{AE}$ and of the inverse simulation are identical to those used in the purely numerical example. The adopted numerical setup is also the same as that described in Sect. 4.1.1. The determined related maximum total energy density, can be seen in Fig. 8a-c. AE to the three pencil-lead breaks located close to Sensors \#3, \#6 and \#7 can be seen. The blue dashed circles indicate the position of the AE sources. The green crosses represent the sensor positions sources (dashed circles) can be identified close to the respective sensors, although some artifacts are still visible. These promising results indicate that TRM can be used for localizing AE sources due to concrete cracking, which is the main aim of this research.

\subsection{Application of TRM to acoustic emissions due to concrete cracking}

Acoustic emissions that were recorded during the double punch test are used as input signals for the inverse simulation. The adopted numerical setup is the same as for the TRM with pencil-lead breaks AE. The results are presented for the four selected AE sets with comparable 


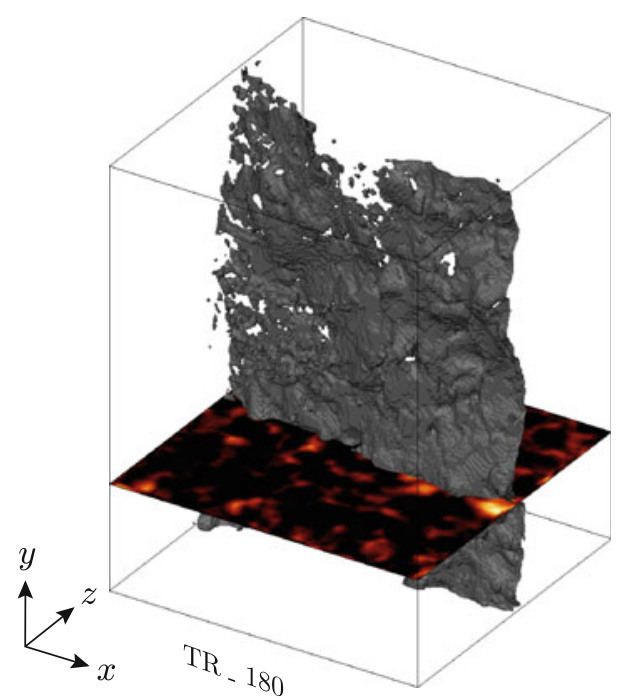

Fig. 9 The related maximum total energy density $\hat{\mathbf{E}}_{\text {tot }}(\mathbf{x})$ at $y=41 \mathrm{~mm}$ is superposed with the threshold-segmented crack volume of the test specimen $(x \times z \times y=120 \times 118 \times 160 \mathrm{~mm})$ after failure. The dominant energy concentrations along the intersection area between the cross-section and threshold-segmented crack indicate the TRM-localized AE due to concrete cracking

input signals, denoted TR_75, TR_82, TR_84 and TR_180, respectively. Fig. 9 shows the related maximum total energy density $\hat{\mathbf{E}}_{\text {tot }}(\mathbf{x})$ resulting from AE set TR_180 for one cross-section that is superposed with the threshold-segmented three-dimensional crack volume of the failed specimen. Note that the four localized AE occurred during crack formation and not in the final stage. After the specimen splits into two parts during the test, almost no energy can be transmitted from one part to the other. From that point onwards, the TRM procedure may no longer be effective. However, in this case the dominant concentration of energy located near the crack indicates a plausible result. It should be noted that the time-reversed $\mathrm{AE}$ sets contain some low frequency components $(0-50 \mathrm{kHz})$, which are caused by the sensors' internal resonance. However, the TRM is used successfully to localize the AE sources. The localized sources of TR_75-180, visible as radiation patterns, are compared to the definite crack distribution from X-ray computed tomography.

The cross-sections presented in Fig. 10a-c exhibit energy concentrations of similar quality. It is interesting to note that in Fig. 10a two sources can be identified. This is not necessarily a conflict, as it is possible that two $\mathrm{AE}$ are released almost simultaneously. Hence, the information of two sources may be contained in one set of recorded displacements. Saenger (2011) demonstrated numerically that two random sources excited at the same time in a three-dimensional, heterogeneous medium can be successfully localized.

\section{Conclusion and outlook}

It is demonstrated with numerical and experimental feasibility studies that the three-dimensional TRM localization performs well for AE released due to concrete cracking. Therefore, from now on TRM can be considered a reliable localization tool in signal-based (a) $\mathbf{T R}$-75

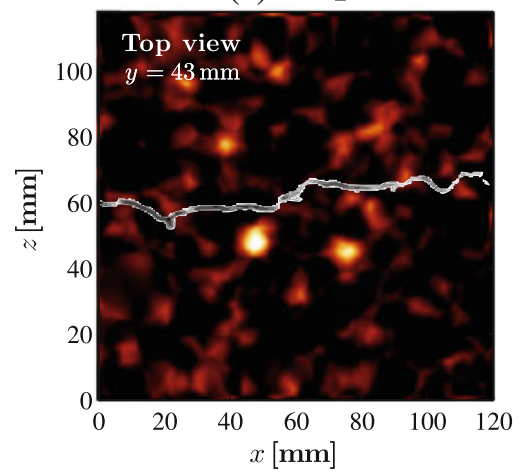

(b) TR_82

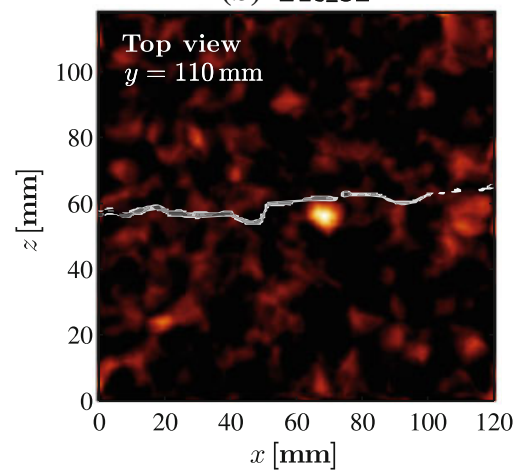

(c) $T R \_84$

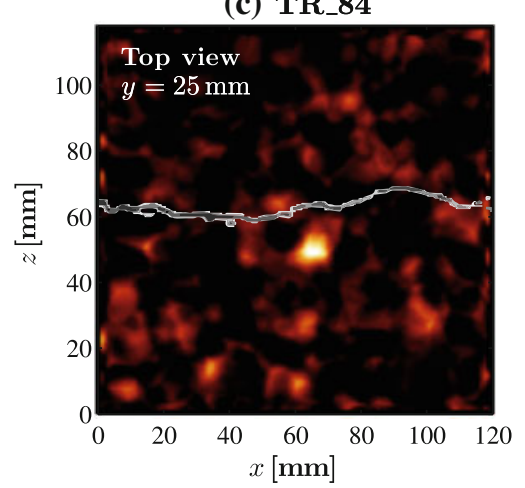

Fig. 10 Specimen cross-sections illustrating the related maximum total energy density $\hat{\mathbf{E}}_{\text {tot }}(\mathbf{x})$ displayed for acoustic emission sets: (a) TR_75 at $y=43 \mathrm{~mm}$, (b) TR_82 at $y=110 \mathrm{~mm}$ and (c) TR_84 at $y=25 \mathrm{~mm}$. The scalar energy field is super- posed with the threshold-segmented crack of the test specimen after failure. The location of the sources in (a)-(c), visible as energy concentrations, agree well with the crack location 
$\mathrm{AE}$ analysis. The dominant concentrations of energy can be well identified, and only a few energy artifacts remain. Further research should focus on the elimination of these artifacts, for example by normalization of signals to provide AE sets of similar energy levels. The instrument response of the sensors needs to be determined, and the recorded $\mathrm{AE}$ signals have to be deconvolved for an optimal application of the TRM. In the current study, the TRM localization was performed for a single set of $\mathrm{AE}$ signals. In commonly used $\mathrm{AE}$ measurements, thousands of AE sets are recorded. It is essential to automate the TRM procedure for an arbitrary number of AE sets. The three-dimensional localization of $\mathrm{AE}$ in concrete specimens can be performed successfully. Applying this method to reinforced concrete members such as beams or slabs is the next logical step. From a theoretical point of view time reverse modeling can be applied to all kinds of elastic materials (as long as the elastodynamic wave equation is valid). Therefore, this approach can also be used for materials other than concrete such as rock or other brittle (or quasi-brittle) and crystalline materials not presented here. However, this method is not necessarily suitable for materials with a high content of air voids, as there may be no energy transmission between neighboring particles. An optimal setup has to be determined for each specific material.

Acknowledgments The authors would like to thank Dr.med. Thomas Frauenfelder from the Institute of Diagnostic Radiology of the University Hospital Zurich for the kind cooperation and for carrying out the $\mathrm{CT}$ scans. Constructive anonymous reviewers have improved the quality of this work. Financial support from the ETHZurich is gratefully acknowledged. E. H. Saenger thanks the DFG (Deutsche Forschungsgemeinschaft) for supporting him through a Heisenberg scholarship (SA 996/1-2).

\section{References}

Achenbach JD (1973) Wave propagation in elastic solids. North Holland, Amsterdam

Chen WF (1970) Double punch test for tensile strength of concrete. ACI Mater J 67(2):993-995
Fink M, Cassereau D, Derode A, Prada C, Roux P, Tanter M, Thomas JL, Wu F (2000) Time-reversed acoustics. Rep Prog Phys 63(12): 1933

Fuller WB, Thomson SE (1907) The laws of proportioning concrete. Trans Am Soc Civ Eng 59:67-143

Grosse CU, Ohtsu M (2008) Acoustic emission testing: basics for research-applications in civil engineering; with contributions by numerous experts. Springer, Heidelberg

Häfner S, Eckhardt S, Luther T, Könke C (2006) Mesoscale modeling of concrete: geometry and numerics. Comput Struct 84:450-461

Kocur GK, Vogel T (2010) Classification of the damage condition of preloaded reinforced concrete slabs using parameter-based acoustic emission analysis. Constr Build Mater 24:2332-2338

Kocur GK, Saenger EH, Vogel T (2010) Elastic wave propagation in a segmented X-ray computed tomography model of a concrete specimen. Constr Build Mater 24:2393-2400

Kurz JH, Grosse CU, Reinhardt HW (2005) Strategies for reliable automatic onset time picking of acoustic emissions and of ultrasound signals in concrete. Ultrasonics 43(7):538546

Marti P (1989) Size effect in double-punch tests on concrete cylinders. ACI Mater J 86(6):597-601

Morse PM, Feshbach H (1953) Methods of theoretical physics: part I. McGraw-Hill Publishing Company, New York

Saenger EH (2008) Numerical methods to determine effective elastic properties. Int J Eng Sci 46:598-605

Saenger EH (2011) Time reverse characterization of sources in heterogeneous media. NDT \& E Int 44(8):751-759. doi:10. 1016/j.ndteint.2011.07.011

Saenger EH, Gold N, Shapiro SA (2000) Modeling the propagation of elastic waves using a modified finite-difference grid. Wave Motion 31(1):77-92

Saenger EH, Kocur GK, Jud R, Torrilhon M (2011) Application of time reverse modeling on non-destructive testing. Appl Math Model 35:807-816

Schechinger B, Vogel T (2007) Acoustic emission for monitoring a reinforced concrete beam subject to four-point-bending. Constr Build Mater 21:483-490

Schubert F, Schechinger B (2002) Numerical modeling of acoustic emission sources and wave propagation in concrete. NDTnet J Nondestr Test 7(9)

Steiner B, Saenger EH, Schmalholz SM (2008) Time reverse modeling of low-frequency microtremors: a potential method for hydrocarbon reservoir localization. Geophys Res Lett 35:L03307

Witten B, Artman B (2011) Signal-to-noise estimates of timereverse images. Geophysics 76(2):MA1-MA10 